\title{
Optimal fits of diffusion constants from single-time data points of Brownian trajectories
}

\author{
Denis Boyer, ${ }^{1, *}$ David S. Dean, ${ }^{2, \dagger}$ Carlos Mejía-Monasterio, ${ }^{3,4, \dagger}$ and Gleb Oshanin ${ }^{5, \S}$ \\ ${ }^{1}$ Instituto de Física, Universidad Nacional Autónoma de México, D.F. 04510, México \\ ${ }^{2}$ Université de Bordeaux and CNRS, Laboratoire Ondes et Matière d'Aquitaine (LOMA), UMR 5798, F-33400 Talence, France \\ ${ }^{3}$ Laboratory of Physical Properties, Technical University of Madrid, Av. Complutense s/n 28040, Madrid, Spain \\ ${ }^{4}$ Department of Mathematics and Statistics, University of Helsinki, P.O. Box 68, FIN-00014 Helsinki, Finland \\ ${ }^{5}$ Laboratoire de Physique Théorique de la Matière Condensée (UMR CNRS 7600), Université Pierre et Marie Curie, \\ 4 place Jussieu, 75252 Paris Cedex 5 France \\ (Received 8 July 2012; published 20 December 2012)
}

\begin{abstract}
Experimental methods based on single particle tracking (SPT) are being increasingly employed in the physical and biological sciences, where nanoscale objects are visualized with high temporal and spatial resolution. SPT can probe interactions between a particle and its environment but the price to be paid is the absence of ensemble averaging and a consequent lack of statistics. Here we address the benchmark question of how to accurately extract the diffusion constant of one single Brownian trajectory. We analyze a class of estimators based on weighted functionals of the square displacement. For a certain choice of the weight function these functionals provide the true ensemble averaged diffusion coefficient, with a precision that increases with the trajectory resolution.
\end{abstract}

DOI: 10.1103/PhysRevE.86.060101

PACS number(s): 05.40.Jc, 31.15.xk, 61.43.Er, 87.16.dp

Single particle tracking (SPT) generates the time series of the position of an individual particle trajectory $\mathbf{B}_{t}$ in a medium (see, e.g., [1,2]). Properly interpreted, the information so obtained provides an insight into the mechanisms and forces that drive or constrain the motion of the particle [3]. Nowadays single particle tracking is extensively used to characterize the microscopic rheological properties of complex media [4] and to probe the active motion of biomolecular motors [5]. In biological cells and complex fluids, SPT methods have become instrumental in demonstrating deviations from standard Brownian motion (BM) [6-10].

The reliability of the information drawn from SPT analysis is not always clear: Data is obtained at high temporal and spatial resolution but at the expense of statistical sample size. Time averaged quantities associated with a given trajectory are subject to large fluctuations across trajectories. For a wide class of anomalous diffusion problems, for instance, time averages of certain particle's observables are, by their very nature, random variables distinct from their ensemble averages [11-14].

Even though standard BM is much better understood than anomalous diffusion processes, averaging problems persist and complicate the analysis of single trajectories. Moreover, in bounded systems, substantial manifestations of sample-tosample fluctuations occur in first passage time phenomena [15]. Standard fitting procedures applied to a finite Brownian trajectory unavoidably lead to fluctuating estimates $D_{f}$ of the diffusion coefficient, due to different thermal histories, particle interactions with different defects, or simply due to blur and localization errors, as discussed in [16-18]. In fact, $D_{f}$ might be very different from the true ensemble average value $D$, as noticed in SPT measurements of diffusion along DNA [19], in

\footnotetext{
*boyer@ fisica.unam.mx

†david.dean@u-bordeaux1.fr

‡carlos.mejia@upm.es

${ }^{\S}$ oshanin@1ptmc.jussieu.fr
}

the plasma membrane [2], or in the cytoplasm of mammalian cells [20].

The broad dispersion of estimate values extracted from common SPT analysis raises an important question: Does an optimal methodology able to determine the diffusion coefficient from just one single trajectory exist? Clearly, it is highly desirable to have an estimator of this kind even for hypothetical pure cases, such as the unconstrained standard BM with perfectly known location at a given time. Such an estimator should possess an ergodic property, i.e., its most probable value should converge to the ensemble average and its variance should vanish as the observation time increases. In addition, knowledge of the distribution of a family of estimators could provide a way to disentangle the effects of the medium complexity or localization errors from variations due to the thermal noise driving microscopic diffusion. Recently it was found that two-time estimates of the diffusion constant for Brownian trajectories with $N$ points can be used to estimate the diffusion constant with a variance $\sim 1 / N$ [16-18].

In this Rapid Communication, we show surprisingly, that among the basic fits using one-time quantities there is a unique fit achieving a variance $1 / \ln (N)$. We focus on determining, when one applies a weighted least-square fit to a BM trajectory, an appropriate weight function which makes the method ergodic. We assume that the Brownian trajectory is known with a given resolution and do not take into account any additional observational error. We study a family of weighted least-squares estimators $\left(u_{\mu}\right)$ of the diffusion coefficient of standard $d$-dimensional $\mathrm{BM}$, given by the following quadratic functionals of a trajectory $\mathbf{B}_{t}$ :

$$
u_{\mu}=\frac{A_{\mu}}{T} \int_{0}^{T} d t \omega(t) \mathbf{B}_{t}^{2},
$$

where $\mathbf{B}_{t=0}=\mathbf{0}, \omega(t)$ is some "trial" weight function of the form

$$
\omega(t)=\frac{1}{\left(t_{0}+t\right)^{\mu}},
$$


$\mu$ being a tunable exponent, $T$ - the observation time, $t_{0}-$ a certain lag time $\left(t_{0} \ll T\right)$, and $A_{\mu}$-the normalization constant. The term "least-squares" and the choice of the weight function will be made clear below.

Here we evaluate the distribution $P\left(u_{\mu}\right)$ for arbitrary $\mu$ and spatial dimension $d$. To easily compare the accuracy of estimators with different values of $\mu$, we chose $A_{\mu}$ such that $\mathbb{E}\left\{u_{\mu}\right\} \equiv 1$, where the symbol $\mathbb{E}\{\ldots\}$ denotes the ensemble average. Hence, $u_{\mu}=D_{f} / D$ with $D$ being the diffusion constant,

$$
D=\frac{\mathbb{E}\left\{\mathbf{B}_{t}^{2}\right\}}{2 d t},
$$

and $D_{f}$-its estimate from $\mathbf{B}_{t}$. The best choice of $\mu$ should produce $P\left(u_{\mu}\right)$ whose maximum $u^{*}$ is the closest to the ensemble averaged value 1 and have the smallest variance $\operatorname{Var}\left(u_{\mu}\right)$. Ultimately, we seek the choice at which $u_{\mu}$ is ergodic, i.e., $D_{f} \rightarrow D$, independently of $\mathbf{B}_{t}$ as $\varepsilon \equiv t_{0} / T \rightarrow 0$.

Before we proceed, two remarks are in order. First, note that $\mu=-1$ corresponds to the standard least-square estimate (LSE) of the square displacement $[2,16,20,21]$. The case $\mu=1$ arises when the unconditional probability of observing the whole trajectory $\mathbf{B}_{t}$ is maximized (assuming that it is Brownian). It is the so-called maximum likelihood estimate (MLE), known to be more accurate than the LSE [16-18,22,23].

We next give a physical interpretation of the estimators in Eq. (1). Consider a least-squares functional:

$$
F=\frac{1}{2} \int_{0}^{T} \frac{\omega(t) d t}{t}\left(\mathbf{B}_{t}^{2}-2 d D_{f} t\right)^{2}
$$

which generalizes the usual functional by adding a time dependent weight function $\omega(t)$ [the standard choice-LSEis $\omega(t) \equiv t]$. The value of $D_{f}$ that minimizes $F$ is

$$
\frac{D_{f}}{D}=\left(\frac{1}{T} \int_{0}^{T} d t \omega(t) \mathbf{B}_{t}^{2}\right) /\left(\frac{2 d D}{T} \int_{0}^{T} d t t \omega(t)\right) .
$$

One recovers Eq. (1) by choosing the weight function $\omega(t)=$ $\left(t_{0}+t\right)^{-\mu}$ and identifying the denominator with $1 / A_{\mu}$. Hence $u_{\mu}$ minimizes a functional (4) and can be referred to as a weighted least-squares estimator. Furthermore, as we discuss below, the continuum limit of Eq. (23) shows that for the function with $\mu=2$ in Eq. (2), the weight function minimizes the variance of $u_{\mu}$.

The moment generating function $\Phi(\sigma)$ of the random variable $u_{\mu}$, Eq. (1), is defined as

$$
\Phi(\sigma)=\mathbb{E}\left\{e^{-\sigma u_{\mu}}\right\}
$$

This function can be calculated using the Feynman-Kac formula (see Refs. [22,23] for more details). For $\mu \neq 2$, we find that to leading order in $\varepsilon=t_{0} / T$,

$$
\Phi(\sigma)=\left[\Gamma(\nu)\left(\frac{\sigma}{\chi_{1}}\right)^{(v-1) / 2} I_{1-v}\left(2 \sqrt{\frac{\sigma}{\chi_{1}}}\right)\right]^{-d / 2},
$$

for $\mu<2$, while for $\mu>2$ it obeys

$$
\Phi(\sigma)=\left[\Gamma\left(1-v\left(\frac{\sigma}{\chi_{2}}\right)^{v / 2} I_{-v}\left(2 \sqrt{\frac{\sigma}{\chi_{2}}}\right)\right]^{-d / 2},\right.
$$

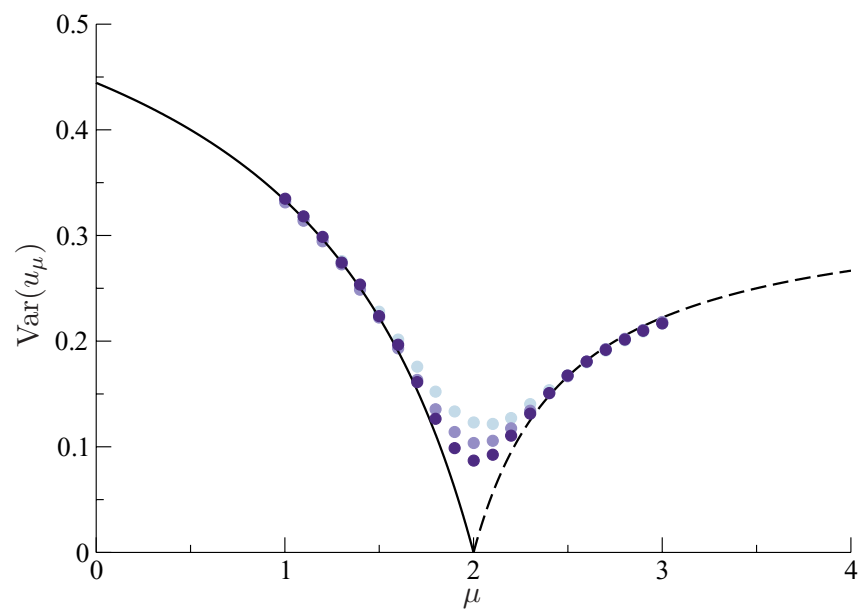

FIG. 1. (Color online) The variance of the distribution $P\left(u_{\mu}\right)$ for different values of $\mu$. The curves correspond to Eq. (9). The symbols correspond to the values obtained from direct numerical simulations of 3D random walks for (from light to dark) $\varepsilon=5 \times 10^{-5}, 5 \times 10^{-6}$, and $5 \times 10^{-7}$.

where $\quad v=1 /(2-\mu), \quad \chi_{1}=d(2-\mu) / 2, \quad \chi_{2}=d(\mu-2) /$ $2(\mu-1)$, and $I_{\mu}(z)$ is the modified Bessel function [24].

The variance $\operatorname{Var}\left(u_{\mu}\right)$ of $P\left(u_{\mu}\right)$ is obtained by differentiating Eqs. (7) or (8) twice with respect to $\sigma$. For arbitrary $\mu \neq 2$ it is then given explicitly by

$$
\operatorname{Var}\left(u_{\mu}\right)=\frac{2}{d} \begin{cases}(2-\mu) /(3-\mu), & \mu<2 \\ (\mu-2) /(2 \mu-3), & \mu>2 .\end{cases}
$$

The consequence of the latter equation is shown in Fig. 1. Unexpectedly, the variance can be made arbitrarily small at leading order in $\varepsilon$ by taking $\mu$ gradually closer to 2 , either from above or from below. The slopes at $\mu=2^{+}$and $\mu=2^{-}$ appear to be the same, so that the accuracy of the estimator will be the same for approaching $\mu=2$ from above or below.

A word of caution is now in order. Finite- $\varepsilon$ corrections to the result in Eq. (9) are of the order $O\left(\varepsilon^{2-\mu}\right)$ for $1<\mu<2$. Therefore the asymptotic behavior above can only be attained when $\varepsilon \ll \exp [-1 /(2-\mu)]$. In other words, the variance can be made arbitrarily small by choosing $\mu$ closer to 2 , but only at the expense of increasing the experimental resolution $\left(t_{0} \rightarrow 0\right.$ or $T \rightarrow \infty)$.

To confirm our analytical results we simulated random walks on a $3 d$ lattice and computed $P\left(u_{\mu}\right)$ using Eq. (1) from a large ensemble of trajectories, for different values of $\mu$ and different resolution $\varepsilon$. For $\mu<1.5$ or $\mu>2.5$, the variance computed numerically is well described by Eq. (9) and is independent of $\varepsilon$ (Fig. 1). Near $\mu=2$, corrections due to the finite resolution are noticeable, but the numerics clearly show that the variance of the distribution $P\left(u_{\mu}\right)$ decreases as $\varepsilon \rightarrow 0$.

The large- and small- $u$ asymptotics of $P\left(u_{\mu}\right)$ can be deduced directly from Eqs. (7) and (8). For $\mu<2$ and $u_{\mu} \ll 1$, $P\left(u_{\mu}\right)$ shows a singular behavior:

$$
P\left(u_{\mu}\right) \sim \exp \left(-\frac{d^{2}}{4 \chi_{1} u_{\mu}}\right) \frac{1}{u_{\mu}^{\zeta}}, \quad \zeta=\frac{3}{2}+\frac{d}{4} \frac{\mu}{|2-\mu|} .
$$




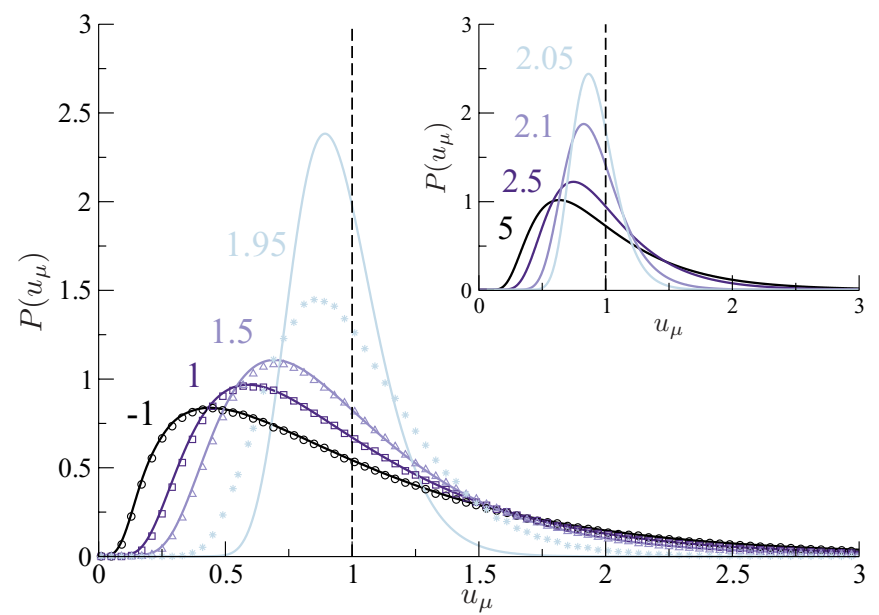

FIG. 2. (Color online) The distribution $P\left(u_{\mu}\right)$ for different $\mu \neq 2$ in $3 \mathrm{D}$ systems. The curves correspond to numerical inversion of Eq. (7) and the symbols to direct numerical simulations of random walks: from dark to light, $\mu=-1$ (circles), $\mu=1$ (squares), $\mu=$ $3 / 2$ (triangles), and $\mu=1.95$ (stars). The numerical values were obtained for $\varepsilon=10^{-5}$, except for $\mu=1.95$ for which we used $\varepsilon=$ $10^{-7}$. Recall that $\mu=-1$ corresponds to LSE and $\mu=1$ to MLE [16-18,22,23]. In the inset we depict the curves corresponding to the inversion of Eq. (8): from dark to light $\mu=5,2.5,2.1$, and 2.05.

The asymptotic behavior for $\mu>2$ can be obtained from Eq. (10) by simply replacing $\chi_{1} \rightarrow \chi_{2}$. Note that Eq. (10) describes a bell-shaped function with a maximal value $u^{*} \rightarrow 1$ when $\mu \rightarrow 2$ from above or below for arbitrary $d$. Next, for $u_{\mu} \gg 1$ and $\mu<2$, we find

$$
P\left(u_{\mu}\right) \sim u_{\mu}^{d / 2-1} \exp \left(-\frac{\chi_{1} \gamma_{1-v, 1}^{2}}{4} u_{\mu}\right)
$$

where $\gamma_{v, 1}$ is the first zero of the Bessel function $J_{v}(z)$ [24]. Results for $\mu>2$ follow from Eq. (11) via the replacements $\chi_{1} \rightarrow \chi_{2}$ and $\gamma_{1-v, 1} \rightarrow \gamma_{-v, 1}$. As $\mu$ gradually approaches 2 , the distribution becomes increasingly narrow: the left tails vanish because of the divergence of the factor $1 /|2-\mu|$ in the exponential, while the right tails vanish because $|2-\mu| \gamma_{-v, 1}^{2}$ and $|2-\mu| \gamma_{1-v, 1}^{2}$ diverge.

The distributions $P\left(u_{\mu}\right)$, obtained by inverting Eqs. (7) and (8), are plotted in Fig. 2. Indeed, the maximal value $u^{*} \rightarrow 1$ when $\mu \rightarrow 2$ either from above or from below. Already for $\mu=1.95$ (or $\mu=2.05$ ) we get the most probable value $u^{*} \approx$ 0.94 , which outperforms the LSE $\left(u^{*} \approx 0.47\right)$ and the MLE $\left(u^{*} \approx 0.6\right)$. For $\mu=1.95$ the variance $\operatorname{Var}\left(u_{\mu}\right) \approx 0.032$, which is an order of magnitude less than the variances observed for $\operatorname{LSE}(=0.5)$ and the MLE $(\approx 0.33)$. Similarly to Fig. 1 , finiteresolution corrections are negligible for $\mu<3 / 2$, and $P\left(u_{\mu}\right)$ is well described by Eq. (7). For $\mu=1.95$ and finite resolution $\varepsilon=10^{-7}$, we obtain a broader distribution and with a smaller $u^{*}$ than that corresponding to Eq. (7) for infinite resolution. However, note that the most probable value of $P\left(u_{1.95}\right)$ that we obtain at finite resolution is $\approx 0.84$, which outperforms the LSE and MLE for infinite resolution.

We turn next to the case $\mu \equiv 2$ with $\varepsilon=t_{0} / T$ small but finite, seeking the variance and the distribution of $u_{\mu=2}$. We consider a slightly more general form for $\omega(t)$ :

$$
\omega(t)= \begin{cases}2 \xi / t_{0}^{2}, & \text { for } t<t_{0} \\ 1 / t^{2}, & \text { for } t_{0} \leqslant t \leqslant T,\end{cases}
$$

where $\xi$ is a tunable amplitude. For such a choice, the moment generating function is given explicitly by

$$
\begin{aligned}
\Phi(\sigma) & =\left(\frac{2 \delta \varepsilon^{(\delta-1) / 2}}{\phi_{+}}\right)^{d / 2}\left[1+\frac{\phi_{-}}{\phi_{+}} \varepsilon^{\delta}\right]^{-d / 2} \\
\phi_{ \pm} & =(\delta \pm 1)\left[\cosh (\sqrt{2 \gamma \xi \sigma}) \pm \frac{\delta \mp 1}{2 \sqrt{2 \gamma \xi \sigma}} \sinh (\sqrt{2 \gamma \xi \sigma})\right]
\end{aligned}
$$

where $\delta=\sqrt{1+4 \gamma \sigma}$ and $\gamma=2 / d[\xi+\ln (1 / \varepsilon)]$. Differentiating Eq. (13), we find

$\operatorname{Var}\left(u_{2}\right)=\frac{4}{3 d} \frac{3 \ln (1 / \varepsilon)-3(1-\varepsilon)+2(1-\varepsilon) \xi+\xi^{2}}{[\xi+\ln (1 / \varepsilon)]^{2}}$.

It is a nonmonotonic function of $\xi$ with a minimum at

$$
\xi=\xi_{\text {opt }}=\frac{(2+\varepsilon) \ln (1 / \varepsilon)-3(1-\varepsilon)}{\ln (1 / \varepsilon)+\varepsilon-1}
$$

The corresponding optimized variance is given by

$$
\operatorname{Var}_{\text {opt }}\left(u_{2}\right)=\frac{4}{3 d} \frac{3 \ln (1 / \varepsilon)-4+5 \varepsilon-\varepsilon^{2}}{\ln (1 / \varepsilon)[\ln (1 / \varepsilon)+1+2 \varepsilon]-3(1-\varepsilon)} .
$$

From Eq. (16) we find that in $3 d \operatorname{Var}_{\text {opt }}\left(u_{2}\right) \approx$ $0.144,0.096,0.082$ for $\varepsilon=10^{-3}, 10^{-5}, 10^{-6}$, respectively. When $\varepsilon \rightarrow 0$, $\operatorname{Var}_{\text {opt }}\left(u_{2}\right)$ vanishes as

$$
\operatorname{Var}_{\mathrm{opt}}\left(u_{2}\right) \sim \frac{4}{d} \frac{1}{\ln (1 / \varepsilon)} .
$$

Therefore, $\operatorname{Var}_{\text {opt }}\left(u_{2}\right)$ can be made arbitrarily small but at the expense of a progressively higher resolution. In the limit $\varepsilon \rightarrow 0$ the distribution converges to a delta function. The estimators with $\mu=2$ are the only ones, in the family defined by Eqs. (1) and (2), that possess an ergodic property. This is shown in Fig. 3 where we plot $P\left(u_{2}\right)$ obtained by numerically inverting Eq. (13) for different resolutions. The symbols in this figure correspond to numerical simulations using the weight function of Eq. (12).

Finally let us consider the case of BM recorded at discrete regular time steps (the calculation can be extended to an arbitrary sequence of time steps [25]) $\Delta j, j=1, \ldots, N$ $(T=\Delta N)$, which is an important problem in its own right but also will allow us to justify the choice of the weight function in Eq. (2). We focus on the estimator of a general form

$$
\tilde{u}=\frac{1}{2 d \Delta} \sum_{j=1}^{N} \omega_{j} \mathbf{B}_{\Delta j}^{2} / \sum_{j=1}^{N} j \omega_{j}
$$

where $\omega_{j}$ now is an arbitrary weight function. The culminating point of our analysis is to determine, via a variational approach, the function $\omega_{j}$ which yields the lowest possible variance $\operatorname{Var}(\tilde{u})$, given from Eq. (18) by

$$
\operatorname{Var}(\tilde{u})=\frac{2}{d} \sum_{j=1}^{N} \omega_{j} \sum_{k=1}^{N} \omega_{k} \min (k, j)^{2} /\left(\sum_{j=1}^{N} j \omega_{j}\right)^{2},
$$




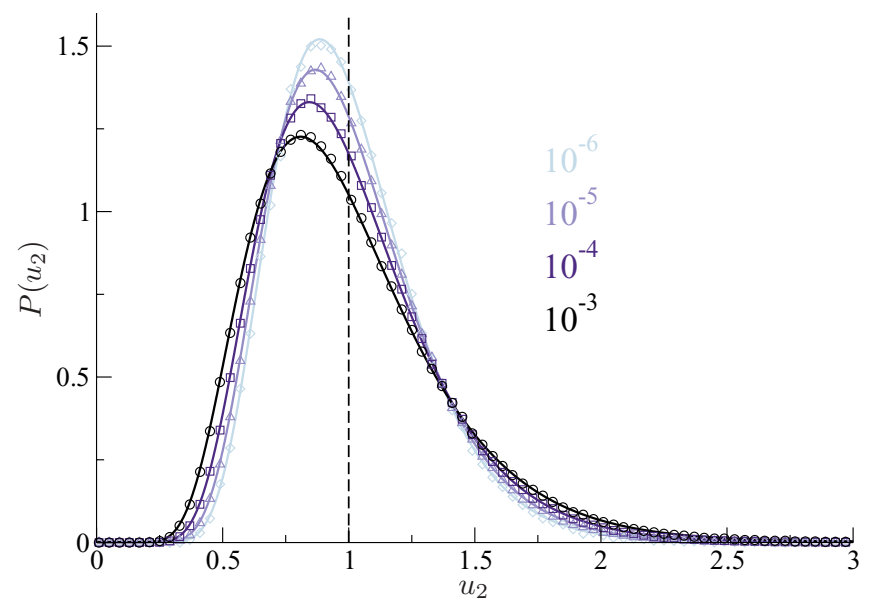

FIG. 3. (Color online) The distribution $P\left(u_{2}\right)$ obtained from a numerical inversion of Eq. (13) for 3D systems. The curves from the dark to light correspond to $\varepsilon=10^{-3}, 10^{-4}, 10^{-5}$, and $10^{-6}$. The symbols are results of numerical simulations of random walks for $\varepsilon=10^{-3}$ (circles), $\varepsilon=10^{-4}$ (squares), $\varepsilon=10^{-5}$ (triangles), and $\varepsilon=10^{-6}$ (diamonds).

where $\min (k, j)$ is the minimum of $k$ and $j$. Minimizing

$$
\tilde{F}=\frac{1}{2} \sum_{j=1}^{N} \omega_{j} \sum_{k=1}^{N} \omega_{k} \min (k, j)^{2}-\lambda\left(\sum_{j=1}^{N} j \omega_{j}-1\right),
$$

with respect to each $\omega_{j}$ ( $\lambda$ is a Lagrange multiplier enforcing the constraint $\mathbb{E}\{\tilde{u}\}=1$ ), we find that the optimal weight obeys

$$
\sum_{j=1}^{N} \omega_{j} \min (k, j)^{2}=\lambda k, \quad k=1, \ldots, N,
$$

which can be solved exactly to give

$$
\lambda=N\left(\sum_{k=1}^{N} \frac{k}{4 k^{2}-1}\right)^{-1}
$$

and

$$
\omega_{j}=\frac{2 \lambda}{4 j^{2}-1}=\left(\frac{N}{\sum_{k=1}^{N} \frac{k}{4 k^{2}-1}}\right) \frac{1}{4 j^{2}-1} .
$$

The optimal variance in this case reads

$$
\operatorname{Var}(\tilde{u})=\frac{1}{d}\left(\sum_{j=1}^{N} \frac{j}{\left(4 j^{2}-1\right)}\right)^{-1} .
$$

Therefore, the weight function in Eq. (23) minimizes the discretized least-squares functional in Eq. (20) and produces an ergodic estimator: the smallest possible variance [for the class of estimators defined by Eq. (18)] vanishes as $N \rightarrow \infty$. Choosing some initial time lag and turning to the limit $\Delta \rightarrow 0$ and $N \rightarrow \infty$, while keeping $\Delta N=T$ fixed, the weight function in Eq. (23) converges to the form in Eq. (2) with $\mu=2$, which thus justifies our choice of the power-law trial weight function for continuous-time Brownian motion. Note that for $N \gg 1$, the leading asymptotic behavior of the variance in Eq. (24) coincides with Eq. (17), but produces slightly higher values of the variance (as the former estimator is based on an everywhere discrete process).

To conclude, we have analyzed the ergodic properties and the asymptotic behavior of a family of least-squares estimators in Eq. (1). We have demonstrated that the estimators with $\mu=$ 2 are the only ones that possess an ergodic property, i.e., they can provide the true ensemble averaged diffusion coefficient from a single trajectory data with any necessary precision, but at the expense of a progressively higher experimental resolution.

We thank Gregory Putzel for valuable comments. The authors acknowledge partial support from the European Science Foundation through the Research Network "Exploring the Physics of Small Devices." C.M.M. is supported by the European Research Council and the Academy of Finland.
[1] Single Particle Tracking and Single Molecule Energy Transfer, edited by C. Bräuchle, D. C. Lamb, and J. Michaelis (WileyVCH, Weinheim, 2010).

[2] M. J. Saxton and K. Jacobson, Annu. Rev. Biophys. Biomol. Struct. 26, 373 (1997).

[3] W. E. Moerner, Proc. Natl. Acad. Sci. USA 104, 12596 (2007).

[4] T. G. Mason and D. A. Weitz, Phys. Rev. Lett. 74, 1250 (1995).

[5] W. J. Greenleaf, M. T. Woodside, and S. M. Block, Annu. Rev. Biophys. Biomol. Struct. 36, 171 (2007).

[6] I. Golding and E. C. Cox, Phys. Rev. Lett. 96, 098102 (2006).

[7] S. C. Weber, A. J. Spakowitz, and J. A. Theriot, Phys. Rev. Lett. 104, 238102 (2010).

[8] I. Bronstein, Y. Israel, E. Kepten, S. Mai, Y. Shav-Tal, E. Barkai, and Y. Garini, Phys. Rev. Lett. 103, 018102 (2009).

[9] G. Seisenberger, M. U. Ried, T. Endreß, H. Büning, M. Hallek, and C. Bräuchle, Science 294, 1929 (2001).
[10] A. V. Weigel, B. Simon, M. M. Tamkun, and D. Krapf, Proc. Natl. Acad. Sci. USA 108, 6438 (2011).

[11] A. Rebenshtok and E. Barkai, Phys. Rev. Lett. 99, 210601 (2007).

[12] J.-H. Jeon, V. Tejedor, S. Burov, E. Barkai, C. Selhuber-Unkel, K. Berg-Sørensen, L. Oddershede, and R. Metzler, Phys. Rev. Lett. 106, 048103 (2011).

[13] Y. He, S. Burov, R. Metzler, and E. Barkai, Phys. Rev. Lett. 101, 058101 (2008).

[14] A. Lubelski, I. M. Sokolov, and J. Klafter, Phys. Rev. Lett. 100, 250602 (2008).

[15] C. Mejía-Monasterio, G. Oshanin, and G. Schehr, J. Stat. Mech. (2011) P06022.

[16] A. J. Berglund, Phys. Rev. E 82, 011917 (2010).

[17] X. Michalet, Phys. Rev. E 82, 041914 (2010); 83, 059904(E) (2011).

[18] X. Michalet and A. J. Berglund, Phys. Rev. E 85, 061916 (2012). 
[19] Y. M. Wang, R. H. Austin, and E. C. Cox, Phys. Rev. Lett. 97, 048302 (2006).

[20] M. Goulian and S. M. Simon, Biophys. J. 79, 2188 (2000).

[21] M. J. Saxton, Biophys. J. 72, 1744 (1997).

[22] D. Boyer and D. S. Dean, J. Phys. A 44, 335003 (2011).
[23] D. Boyer, D. S. Dean, C. Mejía-Monasterio, and G. Oshanin, Phys. Rev. E 85, 031136 (2012).

[24] Handbook of Mathematical Functions, edited by M. Abramowitz and I. R. Stegun (Dover, New York, 1972).

[25] D. Boyer, D. S. Dean, C. Mejía-Monasterio, and G. Oshanin (to be published). 